\title{
Characterization of Shear Strength and Bonding Energy of Laser Pro- duced Welding Seams in Glass
}

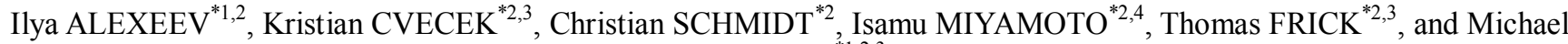 \\ SCHMIDT $^{* 1,2,3}$ \\ ${ }^{* 1}$ Chair of Photonic Technologies, Friedrich-Alexander-University of Erlangen-Nuremberg, Paul-Gordan-Str. 3, 91052 \\ Erlangen, Germany \\ E-mail: ilya.alexeev@lpt.uni-erlangen.de \\ ${ }^{* 2}$ Erlangen Graduate School in Advanced Optical Technologies (SAOT), Friedrich-Alexander-University of \\ Erlangen-Nuremberg, Paul-Gordan-Str. 6, 91052 Erlangen, Germany \\ *3 Bayerisches Laserzentrum GmbH, Konrad-Zuse-Str. 2-6, 91052 Erlangen, Germany \\ ${ }^{* 4}$ University of Osaka, Japan
}

\begin{abstract}
Permanent glass joining can be achieved by an ultrafast laser fusion welding based on nonlinear light energy absorption. In this method ultrashort laser pulses, at a wavelength transparent to the processing material, are tightly focused onto the common interface of two glass pieces and absorbed via nonlinear interaction mechanisms, such as multi photon absorption. In this work we present recent results on the measurements of the shear strength and bonding energy for a number of laser welded soda-lime glass samples produces under different experimental conditions. We demonstrate that the shear strength and the bonding energy are affected differently by the processing parameters and present a qualitative explanation of the observed results.
\end{abstract}

DOI:10.2961/jlmn.2012.03.0009

Keywords: Ultrafast material processing, glass welding, multiphoton processes

\section{Introduction}

Even after hundreds of years of application high quality glass joining remains a challenging technical task. There are a number of methods that are currently used for glass joining such as mechanical mounting, optical contacting, gluing, soldering, and diffusion bonding. Normally they are application specific and possess certain drawbacks: for example, gluing is not suitable for aggressive environments and diffusion bonding requires too long time for many applications. A very promising alternative approach for permanent glass joining can be the ultrafast laser fusion welding based on nonlinear light energy absorption. This method has been recently investigated by a number of research groups that clearly demonstrated method feasibility $[1,2$, $3]$. In comparison to gluing and soldering the ultrafast laser glass welding does not require an additional adhesive. Potentially, it can be applied to non-flat substrates, the latter fundamentally distinguishes the ultrafast laser welding from diffusion bonding, where glass plates are permanently joined under high pressure applied over relatively long time. Compared to the far infrared $\mathrm{CO}_{2}$ laser welding of glass [4], the ultrafast laser joining does not require formation of a vapor-filled capillary, known as "keyhole", for laser energy deposition preserving integrity of the glass surface and reducing the heat affected zone in the material.

In the ultrafast laser glass joining method ultra-short light pulses, at the wavelength transparent to the processing material, are tightly focused onto the common interface of two glass pieces intended for welding. Due to the tight focusing and short laser pulse duration, the light intensity inside the focal volume reaches values at which multiphoton absorption with subsequent ionization can occur [5].
The free electrons generated by this nonlinear interaction become free and are accelerated by absorbing photons directly from the laser beam. These electrons are likely to scatter inelastically on other atoms consequently ionizing some of those atoms and effectively setting off avalanche ionization within the focal volume. Once started, the avalanche ionization can carry on even if the pulse intensity drops below the value necessary for the multiphoton ionization. During the irradiation the electron plasma builds up very quickly and reaches very high temperatures. Subsequently, ions as well as not ionized material are heated by the electron-phonon coupling. In order to reach the melting point of glass it is necessary to have the heating rate higher than the cooling rate. Because the plasma generation takes place approximately within the focal volume $\left(\sim 1-10 \mu \mathrm{m}^{3}\right)$ the cooling time is very short, typically on the order of 10 $\mu$ s or less [6]. Thus, ultrafast glass welding normally requires employment of high repetition rate (on the order of 1 $\mathrm{MHz}$ ) laser systems in order to achieve heat accumulation.

While glass substrates can be welded under different processing conditions (such as laser power, feed speed, pulse repetition rate, and focusing geometry) the mechanical strength of the produced joints varies significantly depending on the applied parameters. For example, use of non optimal processing regimes leads not only to introduction of clearly observable defect structures but also compromises the mechanical strength of the weld seams. In order to quantify the quality of ultrafast laser joining we investigate dependence of the weld seam shear strength and the bonding energy on the processing parameters. The shear strength is used to describe the strength of a joint against the type of structural failure where the weld seam 
fails in shear and can be used for comparison with other well established glass joining methods. Bonding energy is another measure that can be used to quantify the strength of the created connectionss. The bonding energy is work that is needed to separate the glass plates by cleaving, and measured per unit area it can be compared to the surface energy, which is a material constant. In general, surface energy quantifies the disruption of intermolecular bonds that occur when a surface is created

\section{Experimental setup}

Simultaneous requirement on high peak intensity and high pulse repetition rate almost exclusively demands employment of high power solid state picosecond laser systems capable of delivering multi watt ultrafast laser pulses at high frequencies. The light source used in the presented experiments is a modelocked $\mathrm{Nd}: \mathrm{YVO}_{4}$ laser (Fuego laser, Time Bandwidth Products, Switzerland) generating pulses with 10 ps FWHM duration at $1064 \mathrm{~nm}$ wavelength, adjustable pulse repetition rate (single pulse $-8.2 \mathrm{MHz}$ ), and up to $40 \mathrm{~W}$ of nominal average power (continuously adjustable) The schematic of the experimental setup is shown in Fig. 1. The laser beam is focused using an Olympus LMPlan IR 50X / 0.55 microscope objective into the glass samples. The nominal beam focus is positioned slightly (approximately 20 microns) below the common interface of the glass plates constituting a test sample. The welding seam tends to upshift from the nominal laser focus due to the nonlinearity in the light absorption [7] and focusing below the interface ensures the quasi symmetrical laser energy deposition in the upper and lower glass plates. Substantial misplacement of the welding seam above or below the common interface either can prevent samples to be welded all together or can produce very weak joins.

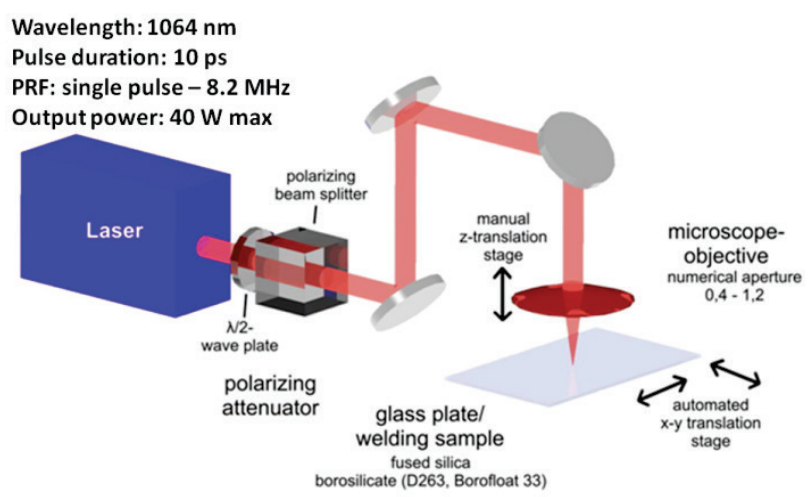

Fig.1: Schematic of the experimental setup.

Before the welding runs the glass plates to be joined are thoroughly cleaned and optically contacted to avoid defect formation during welding and to prevent undesirable relative movement [3]. Additionally, special care is taken to ensure that the laser beam approaches the test sample at the normal incidence angle over its entire area [8] to avoid any longitudinal displacement of the beam focus with respect to the glass interface due to sample tilting.

In the presented work soda-lime glass has been used and the welded samples are analyzed to determine their shear strength and bonding energy. The shear strength is established by measuring the force needed to break the weld seam in the push configuration. The principal test arrangement is shown in Fig. 2. In this setup the welded samples are compressed in the shown direction till the joint is completely compromised yielding the force needed to disassemble the sample. For the testing a universal testing machine has been used and a laser joined sample was held in a vise like holder that restricts its movement without affecting the force measurements. The shear strength is obtained by normalizing the measured force over the welding area. The weld seam dimensions (length and width) are measured with a high resolution optical microscope and for a typical sample the weld seam is on the order of $10 \mathrm{~mm}$ long while the width has characteristic dimension on the order of $50 \mu \mathrm{m}$.

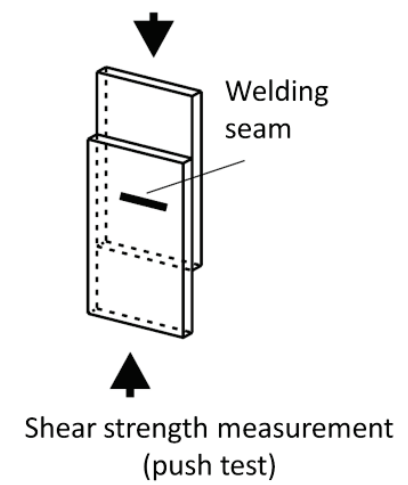

Fig.2: Shear measurement test arrangements.

It should be kept in mind that the optical contact established between the glass plates before welding may also contribute to the shear strength measurement. Although joining due to the intermolecular force is relatively weak, the total effect can be quite noticeable due to the large area of the contact. To exclude contribution of the intermolecular forces from the shear strength measurements, 10 micron thick metal foils have been wedged between the plates near the weld seam to break the optical contact before the compression test. However, special caution has been exhibited not to damage the weld seam. Therefore, a very small portion of the optical contact remained around the welding seam.

To estimate the bonding energy a test setup based on Gillis and Gilman theoretical analysis of crack propagation $[9,10]$ has been assembled with the test schematic being depicted in Fig.3.

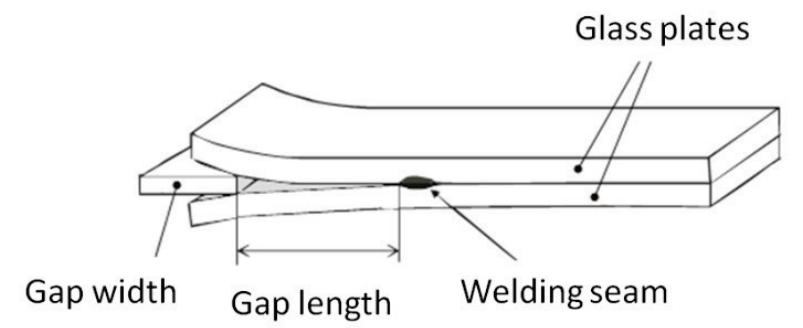

Fig.3: Schematics of the gap opening experiment to determine the bonding energy.

By inserting a blade of thickness $d_{b}$ into the edge of the welded sample and measuring the length of the gap $(L)$ till the joint is destroyed, the bonding energy can be calculated from the following equation: 


$$
E_{b}=\frac{3 E d_{b}^{3} d_{p}^{2}}{32 L^{4}}(1)
$$

where $E$ is the modulus of elasticity of the glass material (Assumed here to be $E=73 \mathrm{GPa}$ ) and $d_{p}$ is the thickness of a single glass plate. It should be pointed out that $E_{b}$ represents the bonding energy of the weld seam itself without the optical contact contribution. For typical gap widths and lengths achieved in this test, the optical contact between the glass plates is broken by the blade well before the weld seam becomes open.

\section{Results and discussions}

The results of the shear strength measurements are shown in Fig.4. For each set of parameters four samples have been produced and results for each test are shown in the figure. Absence of several data points on the chart is due to the sample failure caused by other defects rather than the weld seam breakage. The wide distribution of the measured values within a single processing group is attributed to the material brittleness and presence of defects.

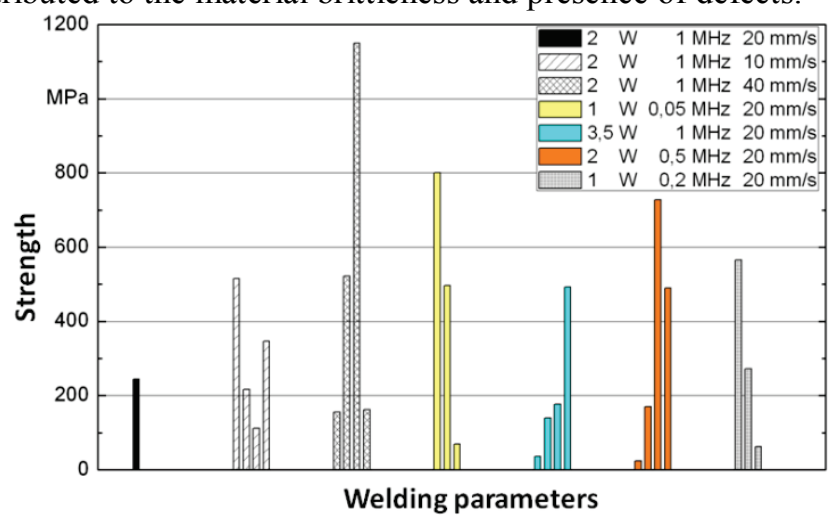

Fig.4: Results of shear strength measurements for different ultrafast laser glass welding parameters.

As it can be seen from the figure, for majority of samples the shear strength varies within 200-600 MPa range. These values are significantly higher compared to the shear strength of other commonly used joining methods: for optical contact bonding $\sim 3 \mathrm{MPa}$ [11], and for high strength adhesive bonding $\sim 29 \mathrm{MPa}$ [12]. At the same time the absolute shear force that can be withstand by the weld seam is relatively low due to the small joining area, which is typically restricted to approximately 50 microns in the transverse direction. The latter may be well acceptable for microjoining where the contact areas are intrinsically small.

Results of the bonding energy measurements are shown in Fig. 5.The experimentally determined bonding energies are in the range from 0.56 to $4 \mathrm{~J} / \mathrm{m}^{2}$ and substantially larger than the bonding energy of the optical contact, which is on the order of $0.1 \pm 0.03 \mathrm{~J} / \mathrm{m}^{2}$. Also the overall mean bonding energy $\left(1.7 \mathrm{~J} / \mathrm{m}^{2}\right)$ is similar to the value determined for the glass plates joined by diffusion bonding [3]. In comparison with the surface energy for soda-lime glass (3.8-3.9 J/m² [13]) the measured value is approximately as twice as small. That points out to a reduced number of bonds that have to be broken relative to the bulk material in order to create a new surface. This is presumably due to the presence of defects in the weld seam.

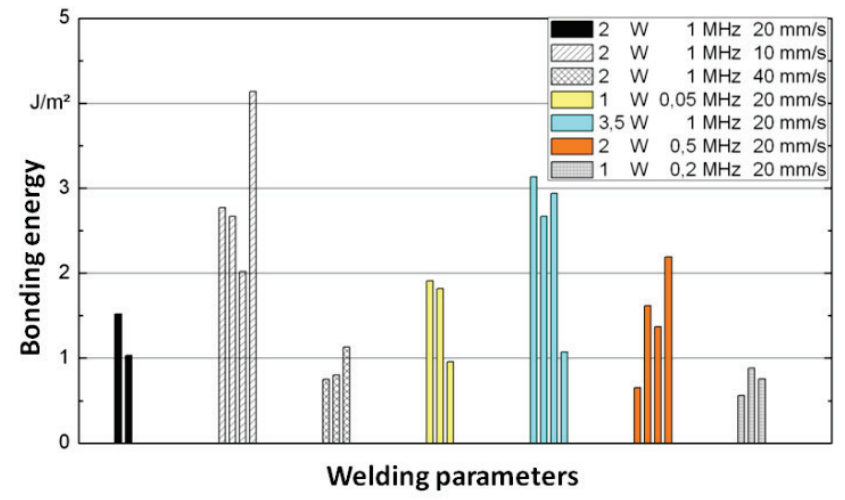

Fig.5: Results for bonding energy measurements for different ultrafast laser welding parameters.

There is an interesting difference in the result distributions for the shear strength and the bonding energy measurements. The bonding energy distribution appears to be more uniform within a single set of parameters compared with the shear strength measurements. This observation can be attributed to the different origins of the sample failure. The failure in shear originates at the weakest point in a structure, which is typically the largest defected produced during the welding process. Since sample-to-sample maximum defect size variation is quite large (even if the samples are produced under identical conditions) the strength test will yield a broad spectrum of values that are best described by Weibull distribution assuming the sample set is relatively large. Meanwhile, the bonding energy rather represents the number of missing intermolecular bonds, which is effectively averaged over all defects in the welding seam resulting in noticeably smaller sample-to-sample variation.

Fig. 6 shows correlation between the mean values of the weld seam strength and the bonding energy for the same set of the processing parameters.

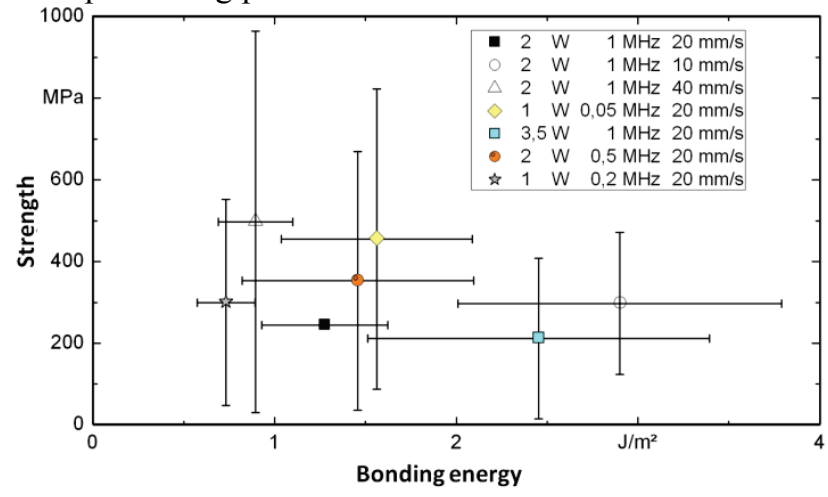

Fig.6: Correlation between the stength and the bonding energy measurements.

Although the error in determination of the mean values is considerably high, a weak trend in the experimental results can be noticed - high strength and high bonding energy of the weld seams seem to move in the opposite directions: high shear strength corresponds to low bonding energy and vise versa. While this counterintuitive result is not completely understood it can be partially correlated with the geometry of the welding volume. The highest bonding energy is achieved at the lowest feeding rate of $10 \mathrm{~mm} / \mathrm{sec}$ that produces the largest interaction volume assuming the other processing parameters are kept constant. In opposite, 
the highest shear strength has been achieved at the fastest feeding rate of $40 \mathrm{~mm} / \mathrm{sec}$ with the same laser power and pulse repetition rate, when the weld seam is significantly smaller. To support this observation the results for the shear strength and the bonding energy measurements are replotted as functions of the weld seam cross section area (in the direction coinciding with the common interface) and are shown in Fig. 7. It has been demonstrated that the latter directly correlates with the volume of the weld seam [14].

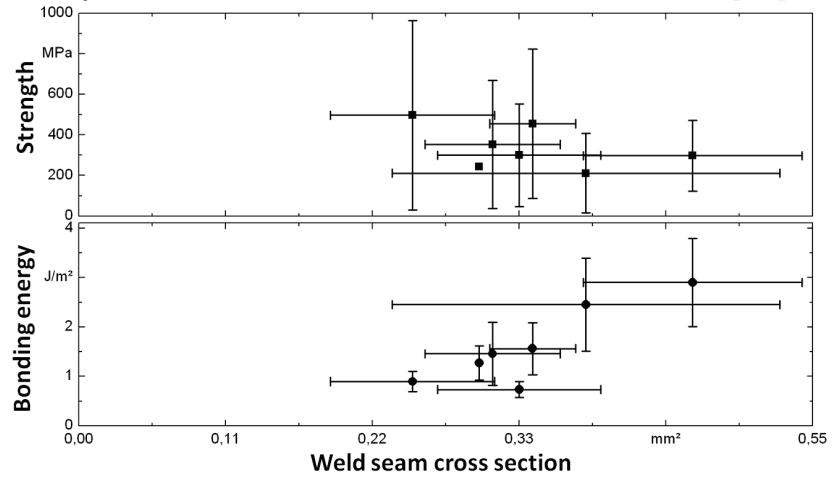

Fig.7: Dependence of the shear strength and the bonding energy measurements on the size of the weld seam.

As it can be seen from the figure, the joint strength is decreasing as the interaction volume is getting larger. This reduction is not due to the volume increase per se but rather due to the fact that larger weld seams tends to have the tear drop transverse shape with a well pronounced sharp tip at the very bottom of the weld redistributing the residual compression stress in the bulk material unevenly (Fig. 8).

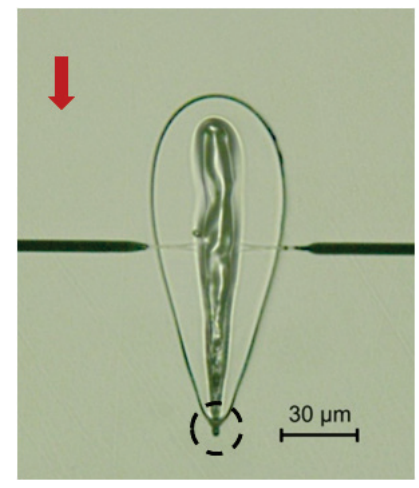

Fig. 8: Exemplary SEM image of a weld seam generated between two D263 glass plates with $4 \mathrm{~W}$ laser power at $1 \mathrm{MHz}$ repetition rate, feed speed $20 \mathrm{~mm} / \mathrm{sec}$, NA 0.55 . The laser beam propagation direction is from top to bottom (red arrow). The dashed circle points to the bottom tip of the molten zone.

Ultimately, the tip can seed the crack propagation undermining the strength of the joint. For smaller interaction volumes the affected region tends to be more symmetrical with a noticeable reduction in the tip size and correspondingly with a reduced seeder crack. Also the residual material stresses around the weld seam have more uniform spatial distribution.

From the chart for the bonding energy it appears that the energy increases with the weld seam area. At this moment such behavior is not completely understood but the following plausible explanation may be proposed. Larger weld seam cross sections and correspondingly larger interaction volumes have longer cool down times. Longer cool down phase allows more efficient material mixing, while glass is in the liquid state, and reduces freezing of micro and nano voids inside the interaction zone. The latter fact promotes establishment of the intermolecular bonds that are normally present in the bulk material increasing the overall bonding energy. While this hypothesis is plausible it still has to be verified. In the authors' opinion this can be done by evaluating the dependence of the total volume of the defects on the total volume of the interaction region by measuring the etching speed.

\section{Conclusion}

In conclusion, we have demonstrated that different merits can be used to quantitatively characterize laser produced weld seams in glass. These merits are the shear strength and the bonding energy that define weld resistance to fail in shear and in cleaving correspondingly. In absolute terms, the achieved shear strength and bonding energy exceed the values typical for conventional joining methods such as gluing or optical contacting, and can be on par with the diffusion bonding. This makes the ultrafast laser glass welding a promising joining technology especially for micro applications. The processing parameters such as the average laser power, pulse repetition rate, and feed rate strongly affect the achievable results and should be carefully selected depending on the application. It has been demonstrated that the above specified merits do not follow each other and optimization of the one may occur at the expense of another. As of now such behavior of the laser produced weld seams is not completely understood but qualitatively attributed to the prevalence of different types of defects generated during the joining process. In this case overall suppression of the defect formation during the ultrafast laser glass welding can lead to higher strength and bonding energy of joints.

\section{Acknowledgements}

This work was partially supported by Erlangen Graduate School in Advanced Optical Technologies, FriedrichAlexander University Erlangen-Nürnberg.

\section{References}

[1] Takayuki Tamaki, Wataru Watanabe, and Kazuyoshi Itoh, Laser micro-welding of transparent materials by a localized heat accumulation effect using a femtosecond fiber laser at $1558 \mathrm{~nm}$, Opt. Express 14, 1046010468 (2006)

[2] I. Miyamoto, A. Horn, J. Gottmann, Local Melting of Glass Material and Its Application to Direct Fusion Welding by Ps-laser Pulses, JLMN-Journal of Laser Micro/Nanoenginneering, Vol. 2, No. 1, p. 7-14 2007

[3] K. Cvecek, I. Miyamoto, J. Strauss, M. Wolf, T. Frick, and M. Schmidt, Sample preparation method for glass welding by ultrashort laser pulses yields higher seam strength, Appl. Opt. 50, 1941-1944 (2011)

[4] Y. Arata, H. Maruo, I. Miyamoto and S. Takeuchi, Dynamic Behavior of Laser Welding and Cutting, Proc. of 7th International Conf. on Electron and Ion Beam Science and Technology, pp. $111-128$, New Jersey (1976)

[5] M. D. Perry, B. C. Stuart, P. S. Banks, M. D. Feit, V. Yanovsky, and A. M. Rubenchik, Ultrashort-pulse la- 
ser machining of dielectric materials, J. Appl. Phys., Vol. 85, No. 9, (1999)

[6] E. G. Gamaly, S. Juodkazis, K. Nishimura, H. Misawa, B. Luther-Davies, L. Hallo, P. Nicolai and V. T. Tikhonchuk, Laser matter interaction in the bulk of a transparent solid: Confined microexplosion and void formation, Phys. Rev. B, 73, 214101, (2006)

[7] Miyamoto, I; Cvecek, K.; Schmidt, M.: Evaluation of nonlinear absorptivity in internal modification of bulk glass by ultrashort laser pulses, Optics Express, Vol. 19, Issue 11, pp. 10714-10727 (2011) http://dx.doi.org/10.1364/OE.19.010714

[8] Schmidt, M; Cvecek, K; Strauß, J.; Alexeev, I.: Focus alignment procedure for high precision processing in transparent media. At: ICALEO 2011, FL, USA.

[9] Peter P. Gillis and J. J. Gilman: Double-Cantilever Cleavage Mode of Crack Propagation, J. Appl. Phys. 35, 647 (1964)

[10] Maszara, W. P.; Goetz, G.; Caviglia, A.; McKitterick, J. B.: Bonding of silicon wafers for silicon-oninsulator, J. Appl. Phys. 64, 4943-4950, (1988)

[11]E J Elliffe et al 2005 Class. Quantum Grav. 22 S257 doi: $10.1088 / 0264-9381 / 22 / 10 / 018$

[12]DELO-PHOTOBOND ${ }^{\circledR}$ GB345 Technical Information, available online at http://www.delo.de/fileadmin/datasheet/DELOPHOTOBOND_GB345_\%28TIDB-GB\%29.pdf

[13] S. M. Wiederhorn, S.M.; Fracture Surface Energy of Glass, J. Am. Ceram. Soc. 52, 99 (1969)

[14]I. Alexeev, K. Cvecek, I. Miyamoto, M. Schmidt "Ultrafast Laser Processing of Transparent Media Glass Welding", Laser in der Elektronikproduktion \& Feinwerktechnik, Tagungsband: LEF 2010, Hrsg.: M. Schmidt, M. Geiger, C. Kägeler, Meisenbach Bamberg 2010

(Received: May 29, 2012, Accepted: August 31, 2012) 